\title{
Metallographische Mitteilungen aus dem Institut für physikalische Chemie der Universität Göttingen.
}

\author{
LXX. \\ Über Magnesium-Siliciumlegierungen. \\ Von \\ R. VOGEL.
}

Mit 1 Figur im Text und 1 Tafel.

Das chemische Verhalten von Silicium zu Magnesium wurde zuerst im Jahre 1858 von Fr. WöHLER ${ }^{1}$ untersucht. Derselbe erhielt durch Erhitzen von Natrium, Chlormagnesium, Fluorsiliciumnatrium und Chlornatrium kleine oktaedrische Krystalle von der Zusammensetzung $\mathrm{Mg}_{4} \mathrm{Si}_{3}$. Im Verfolg der WöHLERschen Arbeit gelangte GeUther, ${ }^{2}$ durch Erhitzen von Fluorsiliciumnatrium mit Magnesium und nachfolgender Entfernung des überschüssigen Magnesiums durch Salmiaklösung zu einem Körper von der Zusammensetzung $\mathrm{Mg}_{5} \mathrm{Si}_{3}$. C. WinkLeR, ${ }^{3}$ welcher Legierungen von der Zusammensetzung $\mathrm{Mg}_{5} \mathrm{Si}_{3}$ und $\mathrm{Mg}_{2} \mathrm{Si}$ durch Erhitzen inniger Gemenge von Siliciumund Magnesiumpulver im $\mathrm{H}$-Strom darstellte, hält $\mathrm{Mg}_{2} \mathrm{Si}$ für die eigentliche, reine Verbindung, $\mathrm{Mg}_{5} \mathrm{Si}_{3}$ dagegen für ein Gemenge mit freiem Silicium. Auf dem Wege der Rückstandsanalyse gelang es endlich neuerdings PaUl Lebead und Robert Bossuet, ${ }^{4}$ aus überschüssigem Magnesium die Verbindung $\mathrm{Mg}_{2} \mathrm{Si}$ zu isolieren.

Die Existenz einer einzigen Verbindung zwischen Magnesium und Silicium von der Formel $\mathrm{Mg}_{2} \mathrm{Si}$ wird, wie hier vorausgeschickt sei, durch das im folgenden beschriebene Zustandsdiagramm der Magnesium-Siliciumlegierungen bestätigt.

1 Ann. Chem. Pharm. Lieb. 107 (1858), 113.

'Journ. pralt. Chem. 95, 424.

"Ber. deutsch. chem. Ges. 23 (1890), 2642.

${ }^{4}$ Compt. rend. 146 (1908), 282. 
Bei den Versuchen, Mg-Si-Legierungen darzustellen, machte sich vor allem die Schwierigkeit geltend, Schmelzgefälse zu finden, welche bei Temperaturen von $1000^{\circ}$ an aufwärts, wie sie zur völligen Auflösung des Siliciums im Magnesium angewandt werden mulsten, nicht mit der Schmelze reagierten. Schmelzgefälse aus Magnesia erwiesen sich oberhalb $900^{\circ}$ wegen ihrer Porosität als ungeeignet, denn die dünnflüssigen Schmelzen wurden von den Rohrwandungen stark aufgesogen und es bildeten sich trotz Überleiten von Wasserstoff, der den Luftstickstoff nicht abzuhalten vermochte, beträchtliche Mengen von Magnesiumnitrid; wurde die so erhaltene Legierung mit Wasser angefeuchtet, so zerfiel sie unter heftiger Erhitzung und Entwickelung von Ammoniak in ein graues Pulver. Versuche mit schwer schmelzbaren Glasrohren, welche sich zur Darstellung von Magnesiumlegierungen, die nicht höher als $800^{\circ}$ erhitzt zu werden brauchen, gut bewährt haben, waren bei der hier erforderlichen Temperatur von mindestens $1000^{\circ}$ aussichtslos. Da. her wurden die Legierungen zunächst in Porzellanröhren hergestellt und ihre Abkühlungskurven bestimmt. Bei vorwiegendem Siliciumgehalt der Legierungen gelang dies ohne grofse Schwierigkeit, während mit wachsendem Magnesiumgehalt das Porzellan so heftig angegriffen wurde, dafs die meisten Versuche infolge Auslaufens der Schmelze oder Zerstörung des Thermoelementes mifslangen. Bei Darstellung der magnesiumreicheren Legierungen wurde bei etwa $1000^{\circ}$ ein vielleicht von der Bildung der Verbindung herrührendes schnelles Ansteigen der Temperatur, sowie ein eigentümlich brodelndes Geräusch bemerkt, während dessen meist das dünnwandige Porzellanschutzrohr des Thermoelementes und dieses selbst zerstört wurde. Analysen ergaben, dafs der Siliciumgehalt dieser in Porzellanrohren hergestellten Legierungen um $10-16 \%$ gestiegen war, was sich aus der reduzierenden Wirkung des Magnesiums auf das Porzellan erklärt. In der Tat war die Wandung der Porzellanschmelzrohre bis auf eine äufsere Schicht in eine dunkle, dem Silicium ähnliche Masse verwandelt. Erhitzt man ferner Magnesium mit zerkleinerten Porzellanstücken in einem Kohlerohr auf $1000^{\circ}$, so findet man bei mikroskopischer Betrachtung der Schliffläche des so erhaltenen Regulus die im Magnesium zerstreut liegenden dunklen Porzellanreste umlagert von den bläulichen Krystallen der Verbindung $\mathrm{Mg}_{2} \mathrm{Si}$. Um festzustellen, dafs die gebildeten Krystalle tatsächlich die genannte Verbindung und nicht etwa ein durch Erhitzen des Magnesiums im Kohlerohr entstandenes Carbid sind, 
wurde Magnesium mit feingepulverter Zuckerkohle in einem Kohlerohr auf $1000^{\circ}$ erhitzt und die Schmelze mit einem Kohlestab durch. gerührt. Nach dieser Behandlung entwickelte das Magnesium mit Säuren allerdings Geruch nach Kohlenwasserstoffen, was auf Carbidbildung schliefsen lälst, dagegen waren der Schmelzpunkt des Magnesiums und das Aussehen der Struktur durchaus unverändert, ein neues Strukturelement hatte sich nicht gebildet.

Die Si-Mg-Gemische wurden daher jetzt in Kohlerohren zusammengeschmolzen und es gelang, Legierungen darzustellen, deren Zusammensetzung mit der nach der Einwage der Metalle zu erwartenden bis auf einen durchschnittlichen Febler von $\pm 1 \%$ übereinstimmte. In das Kohlerohr wurde zurerst das Magnesium in Form eines gerade hineinpassenden zylinderförmigen Stückes eingeführt und das Silicium in linsengrolsen Stücken daraufgelegt. Die Anwendung von Silicium in Form von Pulver oder kleinen Kryställchen erwies sich als ungeeignet und gefährlich, da, vielleicht infolge oberflächlicher Oxydation des Siliciums, seine Vereinigung mit Magnesium erst bei höherer Temperatur und dann sehr heftig erfolgte, wobei es wiederholt vorkam, dafs die Schmelze umhergeschleudert wurde und mit blendendem Licht verbrannte. Die Legierungen von je $10 \mathrm{~g}$ Gewicht wurden unter einer H-Atmosphäre entsprechend ihrem Gehalt an Silicium schnell auf etwa 1000 bis $1500^{\circ}$ erhitzt. Bei Versuchen mit magnesiumreichen Legierungen wurde das Schutzrohr des Thermoelementes berufst und dasselbe erst nach begonnener Abkühlung, kurz vor dem Beginn der Krystallisation in die Schmelze eingetaucht, da auch die Rufsschicht das Porzellanrohr nur kurze Zeit vor der Zerstörung durch die Schmelze schützt. Der Verlauf der Abkühlung der Legierungen wurde von $50-100^{\circ}$ oberhalb des Beginns der Krystallisation (vgl. Fig. 1) bis $500^{\circ}$ verfolgt. Die Temperaturen sind mit Hilfe des Antimon- und Goldschmelzpunktes auf die Skala des Luftthermometers bezogen.

Das zu den Versuchen verwandte Silicium war leider sehr unrein, nach einer Analyse des Herrn Dr. Tamaru enthielt dasselbe $6 \%$ Eisen und $1.7 \%$ Aluminium. In Tabelle 1, welche die den Abkühlungskurven entnommenen Daten enthält, und in dem Zustandsdiagramm der Silicium-Magnesiumlegierungen Fig. 1 sind die Konzentrationen der mit diesem unreinen Silicium hergestellten Legierungen nicht weiter korrigiert. In Fig. 2 beziehen sich die mit Kreuzen bezeichneten Beobach. 
tungen auf Legierungen, welche mit diesem unreinen Silicium dargestellt wurden. Die mit Kreisen bezeichneten Punkte wurden bei Verwendung von reinem $99.2 \%$ igem $\mathrm{Si}$ gefunden.

Tabelle 1.

\begin{tabular}{|c|c|c|c|}
\hline $\begin{array}{l}\text { Mg-Gehalt der } \\
\text { Legierungen } \\
\text { in Gewichtsproz. }\end{array}$ & $\begin{array}{c}\text { Temperatur } \\
\text { des Beginns } \\
\text { der Krystallisation }\end{array}$ & $\begin{array}{l}\text { Temperaturen } \\
\text { der eutektischen } \\
\text { Krystallisation }\end{array}$ & $\begin{array}{c}\text { Krystallisations- } \\
\text { zeiten } \\
\text { in Sekunden }\end{array}$ \\
\hline 0 & \multicolumn{2}{|c|}{$1408^{\circ} \mathrm{Smp}$. d. Siliciums } & 130 \\
\hline 10 & 1335 & $946^{\circ}$ & 45 \\
\hline 30 & 1148 & 945 & 90 \\
\hline 40 & 985 & 950 & 160 \\
\hline 48 & 990 & 950 & 110 \\
\hline$\odot 50$ & 1049 & 969 & 90 \\
\hline 53 & 1067 & 950 & 40 \\
\hline 58 & 1079 & 948 & 30 \\
\hline 63 & 1090 & - & 75 \\
\hline$\odot 63$ & \multicolumn{2}{|c|}{ 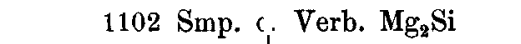 } & 70 \\
\hline 75 & 1052 & 645 & 25 \\
\hline$\odot 81$ & 982 & 658 & 45 \\
\hline 85 & 928 & 642 & 50 \\
\hline 90 & 963 & 645 & 60 \\
\hline 95 & 688 & 645 & .60 \\
\hline 98 & 655 & 646 & 30 \\
\hline 99 & 658 & 646 & 20 \\
\hline 100 & \multicolumn{2}{|c|}{661 Smp. c. Magnesiums } & 100 \\
\hline
\end{tabular}

Wie das Diagramm zeigt, lösen sich Magnesium und Silicium in flüssigem Zustande in allen Verhältnissen und es krystallisiert aus den homogenen Schmelzen zwischen 42 und $96 \%$ Magnesium eine chemische Verbindung von der Formel $\mathrm{Mg}_{2} \mathrm{Si}$. Die Verbindung krystallisiert sowohl mit Silicium als auch mit Magnesium eutektisch. Die Konzentrationen $B$ und $D$ der reinen Eutektika $\mathrm{Si}-\mathrm{Mg}_{2} \mathrm{Si}$ und $\mathrm{Mg}_{2} \mathrm{Si}-\mathrm{Mg}$ liegen bei 42 bzw. $96 \%$ Magnesium. Mischkrystalle treten in den Mg-Si-Legierungen nicht auf, denn die eutektischen Zeiten verschwinden erst bei den reinen Komponenten und auch die Struktur der Legierungen bietet dafür keinerlei Anbaltspunkte. Die Formel $\mathrm{Mg}_{2} \mathrm{Si}$ der Magnesium-Siliciumverbindung ergibt sich aus folgenden Umständen. 1. Die Schmelzkurve hat im Punkte $C$ bei $63 \pm 1 \%$ ein Maximum. 2. Die Eutektika $B$ und $D$ werden Z. anorg. Chem. Bd. 61. 
zufolge Extrapolation der eutektischen Zeiten, bei 63 bzw. $64 \% \mathrm{Mg}$ Null. Hieraus folgt für die Konzentration der reinen Verbindung

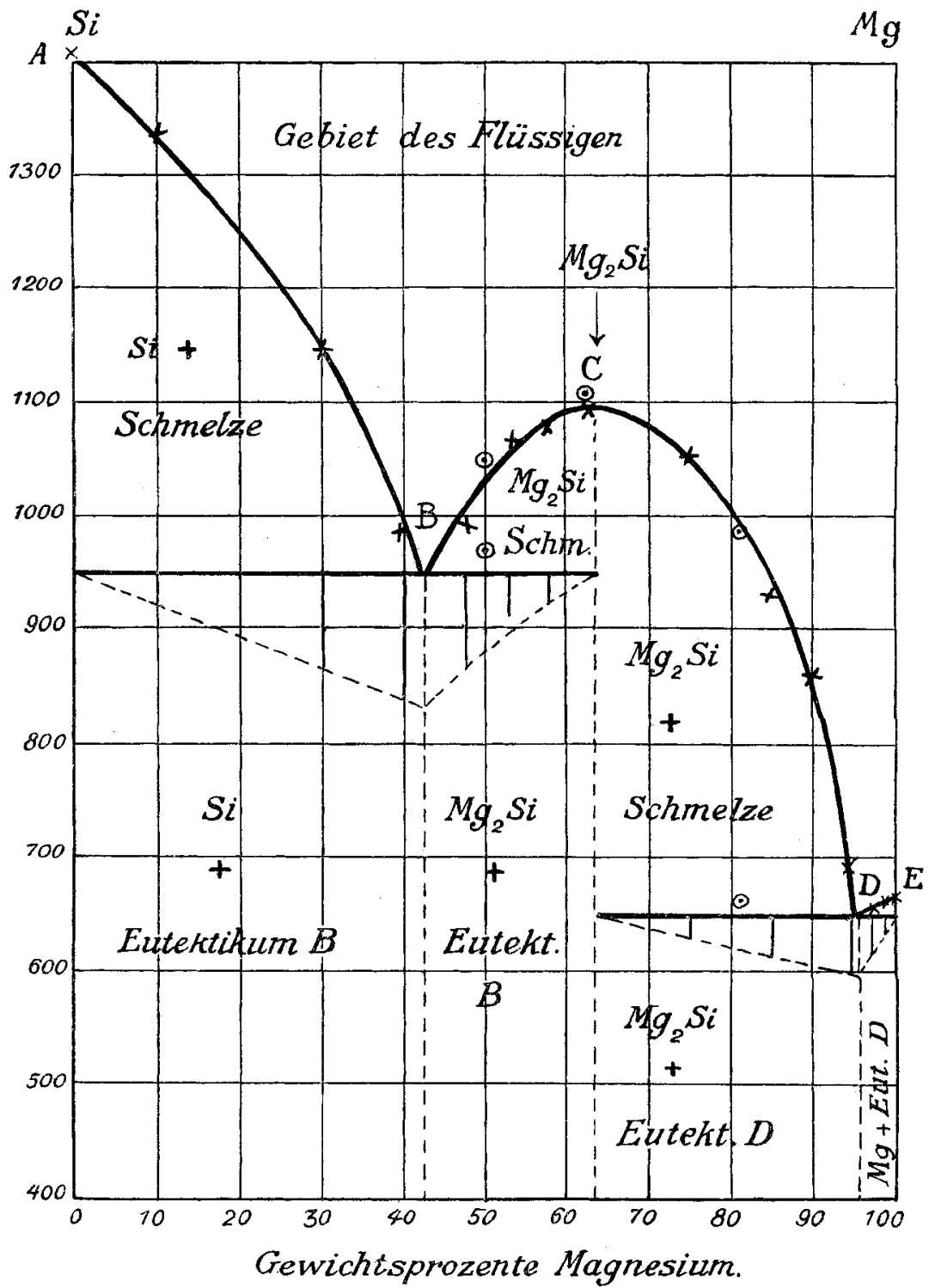

Fig. 1. Zustandsdiagramm der Magnesium-Siliciumlegierungen.

ein Mittelwert von $63.33 \% \mathrm{Mg}$, während die Formel $\mathrm{Mg}_{2} \mathrm{Si}$ $63.17 \mathrm{Mg}$ verlangt. Zur Kontrolle wurde die Verbindung und 
einige benachbarte Konzentrationen mit reinem, 99.2\% igem Silicium, welches auf aluminothermischem Wege bereitet und gereinigt wurde, dargestellt. Diese Versuche ergaben, dafs die Temperaturen des Beginnes und des Endes der Krystallisation bei Verwendung von reinem Silicium ein wenig höher liegen (im Diagramm Fig. 1 durch kleine Kreise bezeichnet). Im übrigen bestätigen sie die vorstehend mitgeteilten Resultate.

Die mit reinem Silicium dargestellte Verbindung $\mathrm{Mg}_{2} \mathrm{Si}$ schmilzt bei $1102^{\circ}$. Sie stellt ein leicht zerbröckelndes Konglomerat glänzender blafsblauer Krystalle dar; dieselben sind ziemlich hart und sehr spröde. An der Luft laufen sie, auch beim Erhitzen, nicht an; von konzentrierter $\mathrm{H}_{2} \mathrm{SO}_{4}$ und $\mathrm{HNO}_{3}$ wird sie nur langsam, schneller von den verdünnten Säuren unter Entwickelung von Wasserstoff angegriffen. Salzsäure dagegen, besonders konzentrierte, zersetzt die Silicidkrystalle anfangs stürmisch unter Entwickelung von selbstentzündlichem Siliciumwasserstoff, darauf lälst die Reaktion bald nach und wird erst beim Erwärmen wieder lebhafter; die nun aufsteigenden Gasblasen entzünden sich nicht mehr. Legierungen, welche aufser der Verbindung überschüssiges Magnesium enthalten, entwickeln mehr, solche mit überschüssigem Silicium weniger selbstentzündlichen Siliciumwasserstoff.

\section{Die Struktur der Legierungen.}

Schon mit blofsem Auge kann man auf den ungeätzten Schliffflächen der siliciumreichen Legierungen die charakteristischen makroskopischen Siliciumnadeln erkennen, welche von den bei zunehmendem Magnesiumgehalt auftretenden oktaedrischen Sicilidkrystallen deutlich verschieden sind. Obgleich die Schmelzen zur Verhütung von Oxydation nicht gerührt worden waren, erwiesen sich alle Reguli als vollständig durchmischt.

Unter dem Mikroskop gewahrt man zwischen $0-42 \% \mathrm{Mg}$ mit wachsendem Magnesiumgehalt eine abnehmende Menge bräunlicher, primär ausgeschiedener Siliciumkrystalle, umgeben von einer wachsenden. Menge des Eutektikums $B$, welches aus bräunlichen Silicium- und bläulichen Silicidlamellen besteht. Fig. 1 (Tafel I) veranschaulicht das Aussehen der Struktur in diesem Gebiet an einer Legierung mit $30 \% \mathrm{Mg}$. Die Schlifffläche ist, um schärfere Kontraste zu erhalten, mit verdünnter Salzsäure geätzt, wobei die grolsen, primären Siliciumkrystalle, sowie die Siliciumpartikel im 
Eutektikum nịcht angegriffen wurden, während der aus der Verbindung bestehende Teil des Eutektikums sich schwarz färbte.

Von $42-96 \% \mathrm{Mg}$ müssen die Legierungen die primär ausge. schiedene Verbindung $\mathrm{Mg}_{2} \mathrm{Si}$ enthalten, von $42-63 \% \mathrm{Mg}$, umgeben von dem Eutektikum $B$, von $63-96 \% \mathrm{Mg}$ von dem Eutektikum $D$. Ätzt man die Schliffe zwischen 42 und $63 \% \mathrm{Mg}$ mit verdünnter Salzsäure, so erkennt man in der Tat (vergl. Fig. 2 Tafel I) das bereits in Fig. 1 gezeigte Eutektikum $B$, in welchem hier dunkel geätzte primär ausgeschiedene Krystalle der Verbindung $\mathrm{Mg}_{2} \mathrm{Si}$ eingebettet liegen. Fig. 3 Tafel I zeigt eine andere, nicht geätzte Stelle desselben Schliffes. Hier erscheint umgekehrt die Verbindung heller und die in Fig. 2 hellen Siliciumlamellen dunkler. Bei $63 \% \mathrm{Mg}$, also nahe der reinen Verbindung $\mathrm{Mg}_{2} \mathrm{Si}$ (welche $63.17 \%$ erfordert) ist das Eutektikum $B$ nicht mehr zu erkennen und eine Legierung von dieser Zusammensetzung, welche praktisch die reine Verbindung darstellt, besteht fast ausschliefslich aus einem lockeren Konglomerat grofser Silicidkrystalle. Vgl. Fig. 4 Tafel I.

Zwischen 63 und $96 \% \mathrm{Mg}$ scheidet sich zum Schlufs der Krystallisation das aus der Verbindung und Magnesium bestehende Eutektikum $D$ aus. Bei geringerem Siliciumgehalt krystallisiert die Verbindung in Form von Skeletten, welche sich ohne Anwendung eines Ätzmittels deutlich von dem hellgelben Grunde des Eutektikums $D$ abheben. Die Kontraste werden schärfer, wenn man die Schliffe durch Einwirkung von Salzsäuredämpfen ätzt. Das vorher hellere Eutektikum $D$ färbt sich dabei dunkler als die Silicidkrystalle und letztere erscheinen hell auf dunklerem Grunde. Die so behandelte Schlifffäche einer Legierung mit $75 \% \mathrm{Mg}$ hat das in Fig. 5 Tafel I wiedergegebene Aussehen. Der Aussage des Diagramms entsprechend sieht man eine überwiegende Menge heller, regelmälsig gelagerter Silicidkrystalle. Die Granulation des sie umgebenden Eutektikums $D$ wird nicht durch Partikel seiner beiden Bestandteile, sondern durch Ätznäpfchen hervorgerufen. Auf der nicht geätzten Schlifffläche einer Legierung mit $90 \% \mathrm{Mg}$, welche nach dem Diagramm nur wenig primär ausgeschiedene Verbindung enthält, sieht man, wie Fig. 6 Tafel I zeigt, grolse dunkle primär ausgeschiedene Silicidskelette, umgeben von dem Eutektikum $D$, dessen Grundmasse reines Magnesium ist und welches zahlreiche kleine Silicidskelette enthält.

In den magnesiumreichsten Legierungen mit $96-100 \% \mathrm{Mg}$ 
findet man das Magnesium als primäre Ausscheidung, umgeben von dem oben beschriebenen Eutektikum $D$.

Silicium verhält sich zu Magnesium ganz ähnlich wie die Elemente derselben natürlichen Gruppe Zinn und Blei, und zwar erstreckt sich diese Analogie nicht nur auf die Formeln der Verbindungen, welche der Vierwertigkeit des Siliciums entsprechen, sondern die Zustandsdiagramme der binären Systeme $\mathrm{Mg}-\mathrm{Si}, \mathrm{Mg}-\mathrm{Sn}$ und $\mathrm{Mg}$ $\mathrm{Pb}$ sind einander aufserordentlich ähnlich.

Herrn Prof. Tammann sage ich für seinen freundlichen Rat und Beistand meinen besten Dank.

Göttingen, Institut für physikalische Chemie der Universität.

Bei der Redaktion eingegangen am 3. November 1908. 